\title{
Designing Warning Interfaces causing Discomfort for Awareness of Risks: Revisited
}

\author{
Ami OTSUKA \\ Tsuda University \\ otsuka@tsuda.ac.jp
}

\author{
Yasuhiro FUJIHARA \\ Hyogo College of Medicine \\ yfuji@hyo-med.ac.jp
}

\author{
Yuko MURAYAMA \\ Tsuda University \\ murayama@tsuda.ac.jp
}

\author{
Tatsuya AOYAGI \\ Tsuda University \\ aoyagi@tsuda.ac.jp
}

\begin{abstract}
Making users aware of the risk by giving them a sense of discomfort and helping them not to access dangerous sites is crucial. Thus, we focus on developing a warning interface, causing discomfort, allowing smartphone users to be aware of danger and risks. We studied discomfort feelings while using smartphones and extracted five discomfort factors from a questionnaire survey and factor analysis. We implemented a prototype of warning interfaces for web browsing on a smartphone considering five factors. In the experiments, we have found that three factors out of the five, namely, "Unintended operation or display," "Sudden changes," and "Understanding of the application," are significant for risk awareness, while the other two are not. This paper reports on the findings of the study.
\end{abstract}

\section{Introduction}

Internet users are exposed to threats such as virus infection, unauthorized access, and phishing scams. These threats are increasing with smartphone use and the spread of IoT technologies. The problem that users are unaware of security threats was pointed out in [1]; however, countermeasures were not considered. Maintaining the awareness of users is crucial to avoid security threats and risks [2]. In this regard, Fujihara et al. [3] surveyed discomfort factors when using personal computers (PCs) and designed risk-aware interfaces based on discomfort feelings. However, the spread of smartphones in recent years has been remarkable. According to "The household ICT device ownership rate [4]" in Japan, smartphone ownership exceeded PC ownership in 2017. The smartphone ownership rate is $83.4 \%$ and the PC ownership rate is $69.1 \%$ in 2019 . Moreover, according to the "Internet usage device by category [4]," 50.4\% used PCs, and 63.3\% used smartphones. The use of smartphones also exceeded that of PCs.
Furthermore, "Attack aimed at smartphones and smartphone applications" was ranked fourth in the "10 Major Security Threats 2018 [5]," in Japan. Moreover, according to McAfee's announcement [6], the threat to mobile devices, and other related things, worldwide has increased suddenly in the second half of 2018.

A smartphone application threat is as follows: Unauthorized and malicious applications steal important information from the device, manipulate the device illegally, and infect the device with ransomware. Various cases occur where malicious apps are installed, hidden in popular applications [6]. When a user browses a website, deceptive sites are trying to input personal information online. Moreover, there are unsafe sites that are damaged by phishing and malware, as well as PCs [7]. In addition, A Symantec's report on mobile threats pointed out that smartphone users are not updated to the latest OS; thus, they are exposed to security vulnerabilities [8]. Human factors are also argued to affect the increased vulnerability of the mobile OS, such as not understanding the security risk due to the old OS version, or being cautious regarding the performance decrease due to the new OS.

Under these circumstances, it is necessary to support user awareness to avoid security threats when using smartphones. In [3], seven factors of discomfort when using a PC were studied, and the authors implemented several warning interfaces [9][10] according to these factors. However, since the discomfort factor when using a smartphone is different from that when using a PC [11], studying an interface when using a smartphone becomes necessary.

The long-term goal of this research is to design a smartphone warning interface that considers the "discomfort feeling" when using a smartphone. This paper reports on a smartphone interface prototype developed for smartphone browsing. 


\section{Related Work}

This section presents risk-aware interfaces that cause discomfort and warnings on smartphones when a user is browsing websites.

\subsection{Risk-aware interfaces}

Human interfaces have been researched extensively in terms of usability [12]. Moreover, studies have also been conducted on methods to avoid human errors in safety engineering. Some interfaces are deliberately designed such that it is difficult to handle the systems that operate them. One example of such systems, which are intentionally developed to be difficult to use, is a blasting dynamite system, designed so that it is not easy to trigger the blast. That is, one has to press two switches simultaneously to initiate the explosion. This type of design has been recommended in military installations [13]. Another example is the fail-safe design of a microwave oven. According to the International Electrotechnical Commission [14], a microwave oven should be designed such that it is not possible to operate it without shutting the door (IEC 60335-2-25).

Such hard-to-use interfaces have also been used in electronic space. When a executes erroneous operations, the system would display a warning message window and ask the user to answer "Yes" or "No" to proceed. However, the problem with this approach is that users tend to answer "Yes" to proceed without completely understanding the warning message.

According to an experimental test conducted by Mackie et al. [15], when the receiver of a message is comfortable, the person would form a reply based on the professionalism of the persuader. In contrast, when the receiver is uncomfortable, the person would form a reply based on the semantics of the message. This study shows that the feeling of discomfort would persuade the user to evaluate decision carefully.

An interface causing discomfort would raise the attention of the user when a warning message is displayed on a computer. For example, some users choose "Yes" without reading warning messages regarding expired server certifications. We consider that the attention of the user to the warning message can be raised by applying discomfort interface principles to the design of the warning. Sankarapandian et al. [16] suggested an interface to make the user aware of the vulnerabilities posed by unpatched software. They implemented a desktop with annoying graffiti that showed the number and seriousness of vulnerabilities. Egelman et al. [17] experimented on the rate to avoid the damage caused by phishing; the experiment was based on a communication-human information processing (C-HIP) model [18], where the interface warns users against the vulnerabilities. They reported that the user responses to a warning differed depending on the type of interface.

\subsection{Smartphone alert interfaces}

As examples of a smartphone alert interface, Nicholson et al. [19] used "nudge," setting the background color or displaying the percentage of people who received the same email in your organization, as an interface to verify the effectiveness of the user's awareness of phishing email. Takahashi et al. [20] pointed out that the conventional alert dialog does not allow the user to delete malware against malware targeting Android OS; thus, they developed a new alert dialog.

For unsafe sites such as phishing sites and malware distribution sites, browsing applications such as Google Chrome and Firefox display warnings [21]. In Google Chrome for Android, when users access unsafe sites with Safe Browsing [22] is enabled, a warning page is displayed explaining the possibility that the website may contain dangerous content, as shown in (Figure 1). Moreover, on the Google search result screen, a warning is displayed next to the site when a site that is not secure is listed in the search result [23].

Security applications also display a warning to a site that is not safe. In the case of Virus Buster Mobile [24] (Trend Micro), a warning page similar to that of Google Chrome, or a pop-up picture with warning messages when tapping a link to the site, is displayed (Figure 1). Protection against threats in in-app browsers is also applied to browsers in most applications.
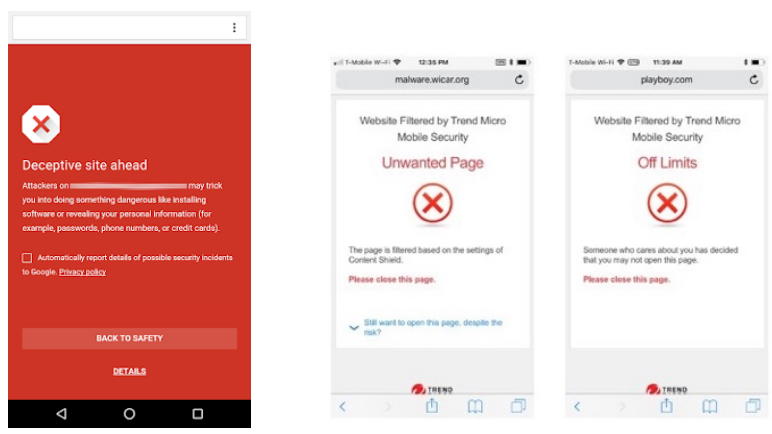

Figure 1. Warning when a user accesses unsafe sites. (left) an warning page, Google Chrome [22], (right) an warning page, Virus Buster Mobile [24]. 


\section{Research Questions}

Our research focuses on considering "discomfort feelings" of the user when using a smartphone to develop a smartphone interface supporting the user's awareness in dangerous situations. The research questions of this study are as follows.

RQ1: Do users actually feel discomfort by the warning interface developed based on discomfort factors when using smartphones?

RQ2: Does the warning interface help users to avoid navigating to unsafe sites?

\section{Methodology}

Previous studies have shown that we conducted a questionnaire survey on user subjectivity and examined the discomfort factors for smartphones through factor analysis. These are described in the first half of this chapter. Subsequently, we implemented a warning interface for web browsing based on five discomfort factors. In the second half of this chapter, we describe the user experiments and interview methods using the interface we have implemented.

\subsection{Discomfort factors among computer system users}

Oikawa [25] identified the factors of discomfort through a questionnaire survey and factor analysis to study the factors causing discomfort among computer system users. They collected 171 discomfort elements from the literature review and a free writing questionnaire survey of 22 undergraduate students (16 males and 6 females). A questionnaire survey and factor analysis were conducted using 86 elements, which were organized into similar from 171 elements. From the analysis results, elements with similar trends in correlation coefficients and factor loadings were combined or removed. In addition, elements that showed high loadings for several factors and elements with questionable basic statistics were excluded, and finally 46 elements were extracted. Using these factors, seven discomfort factors were found from the questionnaire survey and factor analysis and are summarized as follows:

Factor 1) Time consuming Looking for things difficult to find or introducing input information through the keyboard or mouse.

Factor 2) Information seeking Situations where users attempt to find information difficult to locate.
Factor 3) Message

Messages interrupting the activity of the user.

Factor 4) Unexpected operation

System malfunctions that users do not expect or intend.

Factor 5) Difficulty in seeing The sense of sight provided by a physical aspect.

Factor 6) Time delay Wait time and system delays.

Factor 7) Noise

The sense of hearing for a particular sound.

\subsection{Discomfort factors in using smartphone}

Based on the discomfort factors of 46 elements created by studying discomfort when using a computer system, we examined discomfort elements during smartphone use. The typical discomfort elements in smartphone use were modified versions of the wording of the discomfort elements for computer use. For example, "The computer screen suddenly goes dark" was modified to "The smartphone screen suddenly goes dark." The discomfort elements concerning keyboard and mouse operations were corrected to "tap." Thus, we adopted 45 elements from the discomfort elements in computer use. Furthermore, we added eleven elements from a preliminary survey where we asked 18 women undergraduates and graduate students about subjects for comments on situations and events that caused them to feel discomfort in "smartphone use," "Internet use," and "daily life." Finally, we created a questionnaire that consisted of 56 discomfort elements.

We measured the degree of discomfort due to each discomfort element using a questionnaire survey. We asked subjects to rate each discomfort element using five-point Likert scale. The five points scale went from calm (one point) to acute discomfort (five points), and we collected a dataset of 105 elements from women undergraduates and graduate students.

We conducted an exploratory factor analysis on the data and modified the questionnaire. Considering the correlation and relevance between elements after analysis, we excluded 13 elements, and the other 13 elements were gathered into six. We added 11 elements from the preliminary survey that we collected into six elements. Finally, we added four new elements to create 40 question sentences.

We conducted this survey using 40 questionnaire items modified based on the results of the preliminary survey analysis. The survey was conducted between February 15 and February 16, 2018, using a questionnaire system from a survey company's web. Similar to the preliminary survey, our evaluation used a five-point Likert scale. We added to the questionnaire 
three questions related to smartphone use: "smartphone OS (iOS/Android)," "years of use," and "frequently used smartphone functions." To compare this study with [3], we limited the survey subjects to 412 college students (122 males and 290 females). We conducted our analysis on 403 respondents (116 males and 287 females), excluding three people who selected "I do not have a smartphone/I do not use it" in the question regarding the smartphone OS and six people who marked the same rating for 37 of the 40 questions. Among the 403 respondents, 297 were iOS users, and 106 used Android. Regarding the used period, 55 people responded for less than one year; 69 people responded for one to two years; 69 people responded for two to three years; 62 people responded for three to four years, 50 people responded for four to five years; 50 people responded for five to six years; 33 people responded for six to seven years; 13 people responded for seven to eight years. Finally, two people responded for more than ten years.

We performed an exploratory factor analysis for the 403 data points using the maximum likelihood method, Promax rotation. We used IBM SPSS Statistics v23 for the factor analysis. To calculate the average value and the standard deviation value from each question item, we confirmed the ceiling effect for three items: Q1S04 $(M=4.02, S D=1.02), \mathrm{Q} 2 \mathrm{~S} 16(M=4.24$, $S D=1.02)$, and Q2S17 $(M=3.94, S D=1.10)$. Among these three items, we excluded Q2S16, which was unusually high, and reanalyzed 39 items. We conducted our analysis assuming five factors judging from the attenuation state of the eigenvalue obtained from the initial solution and the possibility of interpretation. Thus, we excluded the two items (Q1S07 and Q2S19) for which the loading factor was $<0.300$ and finally conducted factor analysis again on 37 items.

The cumulative contribution ratio considering the total variance of 37 items with five factors before rotation was $48.01 \%$. The items that showed high values for commonality after rotation were Q1S03 (.517) for the first factor, Q2S15 (.519), for the second factor, Q2S17 (.531), and Q2S14 (.504) for the third factor. As Q1S05 (.285) of the first factor and Q2S10 (.278) of the second factor became 0.3 or less, these factors were considered for exclusion; however, they were analyzed from the possibility of interpreting each factor. By examining the reliability of each factor, the Cronbach's alpha for the fifth factor was 0.553, which was somewhat low, but because both were $>0.5$, this was judged as reliable.

We described below the factor name and each feature for the five extracted factors.
Factor 1) Stumbling by system or network Discomfort caused by operation delay or system downtime due to hardware malfunction or poor Internet connection status.

Factor 2) Operation trouble and difficulty in seeing Discomfort due to input and output not performing smoothly.

Factor 3) Unintended operation or display Discomfort due to obtaining unintentional results and performing intended operations.

Factor 4) Sudden changes Discomfort due to extra demands.

Factor 5) Understanding the application Discomfort due to insufficient understanding or inadequate understanding regarding the application use.

The questionnaire survey results regarding feeling discomfort during smartphone use revealed two factors: "Stumbling by system or network" and "Understanding the application." These factors differ from the discomfort factors of computer use. In addition, although we did not extract factors unique to smartphones, all five factors included newly added discomfort elements that were unique to smartphones.

\subsection{Implementation of a Smartphone Interface}

The purpose of this study is to verify whether the five discomfort factors are effective as warnings. Regarding the warning interface verification method, Fujihara et al. [26] developed a "Quiz system with hints" that ran on a PC web browser and evaluated the warning interface for unsafe websites. Moreover, Yamada et al. [27] focused on phishing sites when using smartphones.

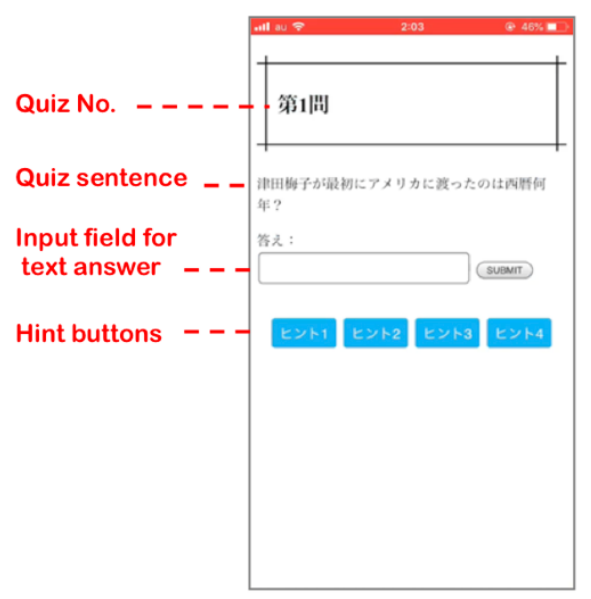

Figure 2. The screen of the quiz application. 

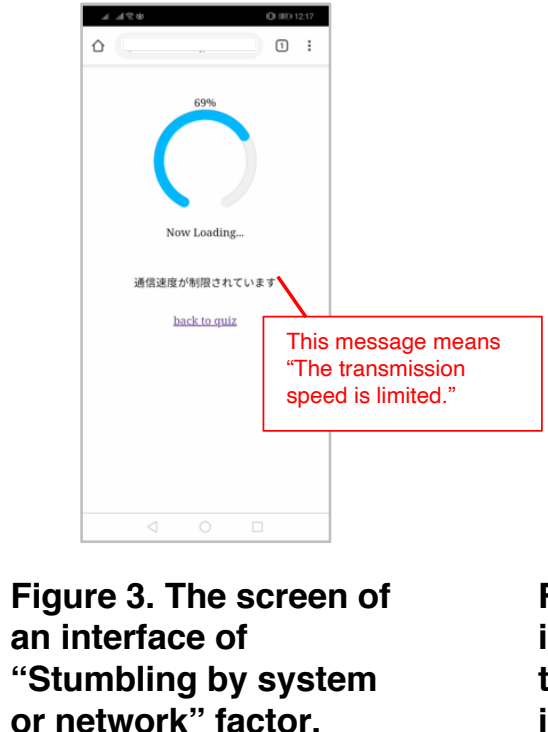
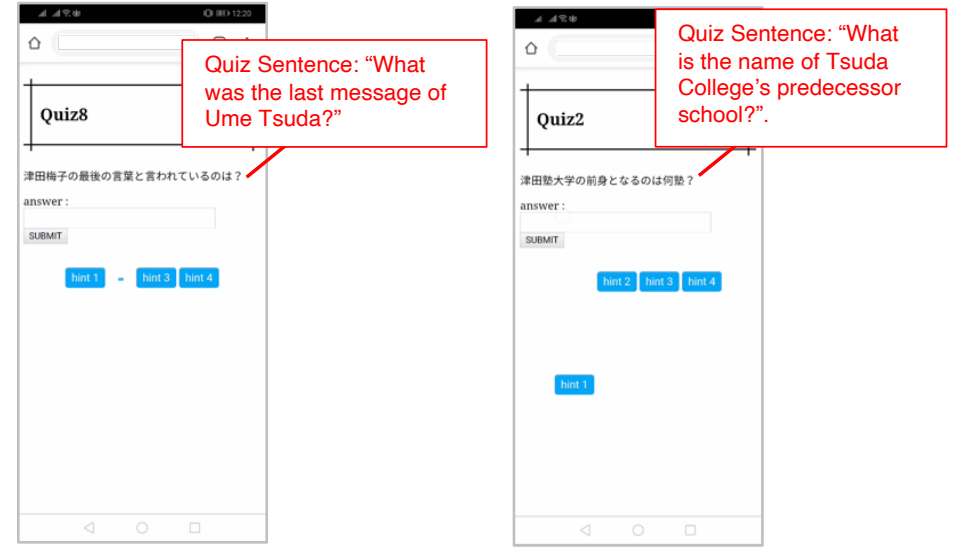

Figure 4. Prototype interface of "Operation trouble and difficulty in seeing" factor.
Figure 5. Prototype interface of "Unintended operation or display" factor.
The authors implemented a warning dialog displayed when entering personal information as a browser application. Finally, they evaluated five login pages. Takahashi et al. [20] evaluated the warning dialog of anti-virus software against malware detection by creating a smartphone game application and incorporating each dialog into it. In this study, we developed a quiz application based on the quiz system with hints from Fujihara et al. [26], considering the quiz system as a warning for browsing and the easiness of developing and verifying all five discomfort factors.

We describe the created quiz application. An example screen of the application is shown in Figure 2. For each question, four hints were prepared as buttons. The three buttons are linked to pages with the correct answer hints, while one button is linked to a page that is assumed to be an unsafe site. When the user taps the hint button to the unsafe site, five warning interfaces are applied randomly. These webpage actions were developed using JavaScript. The warning interfaces using the five discomfort factors are as follows.

\section{Factor 1) Stumbling by system or network}

We can develop interfaces to make users feel aware of factors different from the application, such as operation delays or temporary network shutdowns. If the user tries to tap an unsafe link, a page telling that the connection is delayed due to the speed limit is shown (Figure 3).

\section{Factor 2) Operation trouble and difficulties in seeing}

We can develop interfaces, such as operating range expansion, increasing the number of operations or inputs, and scaling characters more than usual. These interfaces can make it difficult for users to tap the link to the unsafe site by displaying letters in tiny font sizes, requiring time and effort to enlarge the character using two fingers (Figure 4).

\section{Factor 3) Unintended operation or display}

This interface has a button that moves when users try to tap. It cannot be easily tapped even if users make a great effort (Figure 5).

\section{Factor 4) Sudden changes}

This factor indicates that some changes occur on the page. Tapping the link to an unsafe site causes vibration. The vibration time was set to be longer according to the number of taps. The first, second, and third are 3, 5, and $7 \mathrm{~s}$, respectively. For the fourth time, an unsafe site is loaded.

\section{Factor 5) Understanding the application}

This factor indicates that placing buttons in a difficult position to find or understand the application is difficult. Therefore, when the user taps an unsafe site link, another application (e.g., camera, SNS, and calculator) starts functioning. In this experiment, we set up Twitter to run. For the fourth time, an unsafe site is loaded.

\subsection{Experiments and interview}

In this section, we describe the verification method of the warning interface. The study aims to verify whether the users notice that they are trying to access an unsafe site by creating discomfort or whether they avoid accessing the site. Therefore, we conducted experiments 
using quiz apps and interviews. The study was conducted from August 23 to September 11, 2019. To recruit participants for the experiment, we made an announcement to students of the author's university. The announcement stated that 500 yen would be provided as a reward. The participants were fifteen women consisting of undergraduate and graduate students in mathematics and computer science majors (P1, P2, .., P15). The smartphone used in this experiment was HUAWEI Mate 20 lite (Android OS 9.0).

In the experiment, participants were asked to provide a short answer to 10 quiz questions concerning the author's university using hints. Each question was displayed one at a time, and the participants could not return to the previous question to answer it. To encourage the use of hints, we set a quiz with a high difficulty level. We did not explain the warning interface to them before the experiment and told them not to use anything other than the hint pages. During the experiment, the operation screen of the smartphone and the state of the hand were video recorded as videos.

After the experiment, a semi-structured interview was conducted based on the following questions.

1. Did you find any operations or indications you felt or were interested in during the operation? What were the operations and displays? How did you feel when you saw it?

2. (Checking each interface) How did you feel at this display/operation?

3. How do you feel if this type of display or operation occurs on the site, you are browsing when you are searching for something on the Internet with a smartphone?

\subsection{Ethics}

The experiments and interviews were approved by the Independent Ethics Committee of the author's university. All study participants provided informed consent before conducting the experiments and interviews. We reminded to each participant that they could stop the experiment or interview if they feel strong discomfort. Consequently, no one did.

\section{Findings}

First, we found that three interfaces of discomfort factors, "Unintended operation or display," "Sudden changes," and "Understanding of the application," are more likely to avoid the pseudo-unsafe sites than the other two. The number of times that each interface was displayed and the number of times that pseudo unsafe sites were accessed are shown in Table 1. Eighteen cases where the interface did not appear without using hints were excluded. The number of access to a pseudounsafe site in "Stumbling by system or network" and "Operation trouble and difficulty in seeing" is more than half the number of times the interface was displayed, while "Unintended operation or display," "Sudden changes," and "Understanding the application" are less than $20 \%$.

Table 1. The number of times the interface of each discomfort factor was displayed and the number of times participants accessed to pseudo unsafe sites. The number represents the sum of all participants.

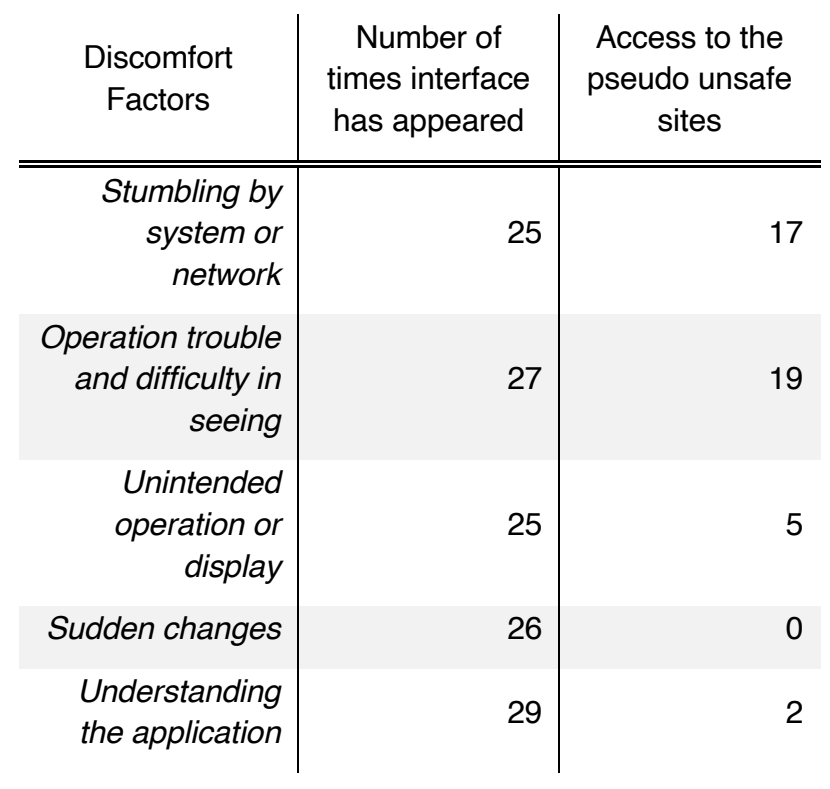

Second, through our interviews, it was found that an interface based on discomfort factors other than "Operation trouble and difficulty in seeing" created anxiety, impatience, and surprise in the participant. Regarding the number of people who answered impressive interface in the first question of the interview (Table 2). There was no significant difference at each interface.

Some of the opinions from the interview about each interface are as follows.

Regarding the interface of Factor 1) Stumbling by system or network, some comments seemed discomfort; "I got impatient (P1).", "I thought I was going to another site and came right back (P3).", "I felt like I was accessing a dangerous ad (P10)." Moreover, some participants said that they were waiting for the loading display (\%) to progress (P7), that they were waiting for a hint (P5, P8, P9, P11), and that they were not aware of the communication limitations and thought it was just a 
Table 2. Number of people who answered impressive regarding each interface.

\begin{tabular}{l|r} 
Discomfort Factors & Number of people \\
\hline $\begin{array}{l}\text { Stumbling by system or } \\
\text { network }\end{array}$ & 11 \\
$\begin{array}{l}\text { Operation trouble and } \\
\text { difficulty in seeing }\end{array}$ & 8 \\
$\begin{array}{l}\text { Unintended operation } \\
\text { or display }\end{array}$ & 10 \\
$\begin{array}{l}\text { Sudden changes } \\
\text { Understanding the } \\
\text { application }\end{array}$ & 8 \\
\end{tabular}

loading situation (P1, P4). P6 stated, "I thought it was an access bug."

Concerning the interface of Factor 2) Operation trouble and difficulty in seeing, most participants indicated that they did not think it was a special interface, as shown below; "I did not notice the button itself. (P1, P3, P10, P11, P13, P15).", "I thought it was a bug or loading failure (P1, P4, P6, P10, P12).", "I did not think I could tap (P11, P13)."

Regarding the interface of Factor 3) Unintended operation or display, most participants said, "I thought it was meaningful (P3, P8, P9, P11)." P5 mentioned, "I felt anxiety," and P2 mentioned, "It felt unpleasant," P4 and P5 said, "I was surprised." There were some symmetrical comments, such as "I thought a few presses would give me a hint (P8, P12)" and "I did not think I would be able to press it more than once (P13)." Other comments were also obtained, "I thought it was a bug (P6, P7)" and "It was interesting, and I pushed many times (P7)."

Regarding the interface of Factor 4) Sudden changes, the most common comment was, "I thought it was a notification for an incorrect answer or another app (P1, P3, P4, P5, P13)." Other comments were, "I was surprised (P4, P8, P9).", "I felt anxiety (P4, P5)," and "I got impatient(P10)."

Finally, considering the interface of Factor 5) Understanding the application, the most common comment was, "I was surprised (P2, P8, P10, P11, P13)." Some participants expressed security concerns, "I thought my personal information was stolen (P3, P10)" and "Suspected phishing (P6)." There were some symmetrical comments, such as "I thought I would get a hint if I logged in, but I got lost (P4, P7, P13)," and "I did not log in because I did not think it would be a hint
(P9, P14)." Other opinions were, "It was frustrating (P1)," and "I thought it was malfunctioning (P8)."

\section{Discussion}

Excluding "Operation trouble and difficulty in seeing," four interfaces produce desirable feelings as discomfort interfaces, such as anxiety, impatience, surprise, and disgust. Moreover, the answers included keywords related to security risks such as viruses, unauthorized access, and phishing. Furthermore, according to the number of people who answered impressively at each interface, no significant difference seems to be present in terms of impression for each interface. However, at the interface of "Stumbling by system or network," pseudo unsafe sites were accessed at a rate of $70 \%$. Therefore, it is considered that the interfaces of three factors, namely, "Unintended operation or display," "Sudden changes," and "Understanding of the application," may be effective in risk awareness.

Subsequently, we discuss each interface. First, for the interface of "Stumbling by system or network" factor, there were opinions such as "I was waiting because the reading display (\%) was progressing (P7)," and "I think the loading page that I often see (P10)." As an improved measure, we are considering following an approach similar to a gasoline meter.

For the interface of "Operation trouble and difficulty in seeing," the factor could not trigger the pinch movement that we intended but also the opinions, "I did not notice the button itself (P1, P3, P10, P11, P13, $\mathrm{P} 15), "$ and "I thought it was a bug or loading failure (P1, P4, P6, P10, P12)". Therefore, it is necessary to consider improving the interface.

Finally, for the interface of "Understanding the application" factor, as multiple opinions regarding the input of personal information arose, it is necessary to verify this factor by using other applications less relevant to the quiz app.

The survey was limited to university students as they are the generation that most frequently uses smartphones. In addition, because the study was conducted and experimented with women, gender bias could be present.

\section{Conclusion}

We conducted user experiments and interviews related to warning interfaces for web browsing based on five factors of discomfort for smartphone users. The purpose of this experiment was to test whether interfaces based on the proposed five discomfort factors actually cause discomfort to users and whether they are 
effective for risk awareness. Consequently, it was suggested that the interfaces of "Unintended operation or display," "Sudden changes," and "Understanding of the application" are prone to be effective in risk awareness. Using these interfaces and existing alerts, we plan to conduct comparative experiments on user behavior in response to alerts.

For the future, we need to consider the issue of familiarization, and the state of the user before and after the warning is displayed. We also will explore the possibility of applying the discomfort-based interface to other fields different from warnings.

\section{References}

[1] Murayama, Y., Hikage, N., Hauser, C., Chakraborty, B. and Segawa, N. (2006). An Anshin model for the evaluation of the sense of security, Proc. Of the $39^{\text {th }}$ Hawaii International Conference on System Science (HICSS'06), (Vol. 8, p. 205a).

[2] Dourish, P. and Bly, S. (2011). Portholes: Supporting awareness in a distributed work group, Proc. SIGCHI Conference on Human Factors in Computing Systems, pp. 541-547.

[3] Fujihara, Y. and Murayama, Y. (2011). A proposal of warning interfaces causing discomfort for awareness of security threats and human errors, Journal of Information Processing Society of Japan, 52(1), pp. $77-$ 89.

[4] Ministry of Internal Affairs and Communications (2020). WHITE PAPER Information and Communications in Japan, Part 2, Chap.5, Section 2-1. https://www.soumu.go.jp/johotsusintokei/whitepaper/j $\mathrm{a} / \mathrm{r} 02 / \mathrm{html} / \mathrm{nd} 252110 . \mathrm{html}$ (accessed 2020-11-10)

[5] Ten Major Security Threats 2019 (2019). Informationtechnology Promotion Agency, Japan (IPA). https://www.ipa.go.jp/files/000072668.pdf (accessed 2019-06-15)

[6] McAfee Mobile Threat Report Q1. 2019 https://www.mcafee.com/enterprise/enus/assets/reports/rp-mobile-threat-report-2019.pdf (accessed 2019-06-15)

[7] Google Chrome Help, manage warnings about unsafe sites

https://support.google.com/chrome/answer/99020?co= GENIE.Platform $\% 3$ DAndroid $\& \mathrm{hl}=\mathrm{en} \&$ oco $=0$ (accessed 2019-06-15)

[8] Symantec mobile threat intelligence report 2017. The Year In Review,

https://www.symantec.com/content/dam/symantec/doc s/reports/mobile-threat-intelligence-report-2017-en.pdf (accessed 2019-06-15).

[9] Fujihara. Y., Murakami, H., Kanamori, Y., Saito, Y., and Murayama, Y. (2009). An implementation of an interface causing discomfort for awareness of risks and threats on web browsing, IPSJ Symposium Series (DICOMO'09), (Vol. 1 pp. 85-91) .

[10] Fujihara, Y., Kanamori, Y., Mukai, M. and Murayama, Y. (2010). An interface causing discomfort to prevent user from missending e-mail messages to incorrect addresses, Poster and Demonstration Paper, Proc. 4th IFIP WG 11.11 International Conference on Trust Management (IFIPTM 2010), pp. 13-16 .

[11] Otsuka, A., Fujihara. Y., Murayama, Y., Aoyagi, T. (2019), Analysis of discomfort factors in smartphone use, Proc. Of the $52^{\text {th }}$ Hawaii International Conference on System Science (HICSS'19).

[12] Nielsen, J. 1993. Usability Engineering. Academic Press.

[13] Norman, D.A. 1988. The Psychology of Everyday Things. Basic Books.

[14] International Electrotechnical Commission 1996. Safety of household and similar electrical appliances part 2: Particular requirements for microwave ovens (MOD IEC 60335-2-25)

[15] Mackie, D. M. \& Worth, L. T. (1989). Processing deficits and the mediation of positive affect in persuasion. Journal of personality and social psychology, 57(1), pp. 27-40.

[16] Sankarpandian, K., Little, T. and Edwards, W.K. (2008). TALC: using desktop graffiti to fight software vulnerability, Proc. ACM CHI 2008 Conference on Human Factors in Computing Systems, pp. 1055-1064.

[17] Egelman, S., Cranor, L. F., and Hong, J. (2008). You've been warned: An empirical study of the effectiveness of web browser phishing warnings. Proceedings of $A C M$ CHI 2008 Conference on Human Factors in Computing Systems, pp. 1065-1074.

[18] Wogalter, M. S. (2006). Communication-Human Information Processing (C-HIP) Model. In Wogalter,M.S.(Ed) Handbook of Warnings. Lawrence Erlbaum Associates, 51-61.

[19] James Nicholson, Lynne Coventry, and Pam Briggs. (2017). Can we fight social engineering attacks by social means? Assessing social salience as a means to improve phish detection. Thirteenth Symposium on Usable Privacy and Security (SOUPS 2017) (pp. 285298).

[20] Takahashi, A., Takada, T., (2015), Designing Effective Alert Dialog to encourage Android Users to take Countermearsure Action to Android Malware, Journal of Information Processing Society of Japan, 56(12), pp.2302-2312.

[21] Mozilla Firefox for Android, Mixed content blocker in Firefox for Android (accessed 2019-06-15) https://support.mozilla.org/en-US/kb/mixed-contentblocker-firefox-android

[22] Google Safe Browsing https://developers.google.com/safe-browsing/ (accessed 2019-06-15)

[23] Google Transparency Report https://ransparencyreport.google.com/safebrowsing/overview (accessed 2019-06-15)

[24] Trend Micro VirusBuster Mobile https://blog.trendmicro.com/vpn-101-part-2-set-vpncontent-shield-trend-micro-mobile-securityios/(accessed 2020-09-30)

[25] Oikawa, H. (2008). A study of a causal structure model for a discomfort interface, Master's thesis, Iwate Prefectural University Graduate School. 
[26] Fujihara. Y., Murakami, H., Kanamori, Y., Saito, Y., Murayama, Y., (2009). An implementation of an interface causing discomfort for awareness of risks and threats on web browsing, IPSJ Symposium Series (DICOMO'09), (Vol. 1 pp. 85-91)

[27] Yamda, K., Ogura, K., Bista, B., and Takata, T. (2017). A Study of alert dialog to raise security awareness considering restriction by screen size of smartphone, SIG Technical Reports, 2017-HCI-172, 21, pp. 1-8.

\section{Appendices}

\section{Question Items}

Q1S01) It takes time to start-up or shut down or restart the smartphone.

Q1S02) The operation of the smartphone is slow because processing that is beneficial to you, such as virus search and update.

Q1S03) The operation is slow because the performance of the smartphone is not good.

Q1S04) The smartphone suddenly restarts or stops moving.

Q1S05) The screen of the smartphone suddenly becomes dark.

Q1S06) If an error message is displayed for the operation performed, you cannot complete the operation.

Q1S07) A confirmation message appears each time you perform a specific operation.

Q1S08) A message notifying the update of software (application) is displayed during work.

Q1S09) Software (application) is updated and installed without permission.

Q1S10) The application was unintentionally started (calling, the camera was running, etc.)

Q1S11) You need to use or install another application to use certain functions in the application.

Q1S12) It is difficult to understand how to use the application.

Q1S13) It is necessary to find or switch the desired application with multiple applications open.

Q1S14) It is difficult to find applications and files that you want to use.
Q1S15) Suddenly big sounds and movies are played from the smartphone.

Q1S16) A suddenly vibration is transmitted from the smartphone

Q1S17) Fingerprint authentication or face authentication does not respond

Q1S18) The battery of the smartphone is about to run out when going out.

Q1S19) It takes time to display pages on the web or in the application.

Q1S20) You can not connect to the Internet.

Q1S21) There is communication restriction or speed limit.

Q2S01) It is difficult to determine what kind of information is on the screen.

Q2S02) You do not know if the information posted on the web page is accurate.

Q2S03) Ads are displayed on the screen.

Q2S04) It is difficult to see the background of the web page and the color of the text.

Q2S05) You see sentences or images that make you feel bad.

Q2S06) You read small size letters.

Q2S07) You read a long sentence and a web page that has many contents and is vertically long.

Q2S08) You enter a long URL (web page address).

Q2S09) You were asked for ID or password.

Q2S10) You forget the necessary password.

Q2S11) When registering personal information, there are many input items.

Q2S12) Entry of information that you do not want to enter when entering personal information is included in essential items.

Q2S13) Kanji conversion is not done as desired.

Q2S14) You can not tap the place you want.

Q2S15) It is difficult to copy and paste.

Q2S16) Ads are displayed where you want to tap.

Q2S17) Unintentionally tapping ads.

Q2S18) It is necessary to pinch with two fingers or multi-touch (operate with two or more fingers simultaneously). 